\title{
Protective effect of quercetin on weight drop injury model-induced neuroinflammation alterations in brain of mice
}

\author{
Hmangte Hitler Kom, Mesram Nageshwar, Kota Srilatha, Karnati Pratap Reddy* \\ Department of Zoology, Osmania University, Hyderabad, India.
}

\section{ARTICLE INFO \\ Received on: 14/12/2018 \\ Accepted on: 06/03/2019 \\ Available online: 18/04/2019}

\section{Key words:}

Quercetin, weight drop injury, neuroinflammation, oxidative stress, histological alteration.

\begin{abstract}
The neuroinflammatory response following traumatic brain injury is known to be a key secondary injury factor that can play a critical role in accelerating the progression of neurodegeneration. This study reports protective effect of dietary quercetin against neuroinflammation-induced changes in mice triggered by weight drop injury model. Quercetin is a natural flavonoid found in high quantities in fruits and vegetables, with properties of antioxidant, free radical scavenger, and anti-inflammation. Hence, this study reports the effects of quercetin on neuroinflammation-mediated oxidative stress and behavioral and histological alterations in mice. Mice were divided into four groups: control, inflammation, inflammation + Quercetin (20 mg/kg mass), and Quercetin (20 mg/kg mass). The treatments were carried out on mice for 7 days and subsequently, behavioral studies were conducted and then brains were collected and used for biochemical and histological studies. Mice undergoing inflammation showed significant $(p<0.05)$ deficits in motor coordination, increase in the paw withdrawal latency period, and levels of lipid peroxidation, as well as decline in the activities of antioxidant enzymes, such as superoxide dismutase, catalase, glutathione peroxidase, and histological alterations in cerebral cortex region in the brain compared with the control group. Quercetin treatment significantly $(p<0.05)$ reversed the behavioral alterations, decreased the lipid peroxidation level, and increased the superoxide dismutase, catalase, glutathione peroxidase activities, and histological alterations in cerebral cortex region of the brain compared with the inflammation group. These findings suggest that dietary quercetin has potential benefits in mitigating neuroinflammation-mediated oxidative stress and behavioral and histological alterations.
\end{abstract}

\section{INTRODUCTION}

Neuroinflammation is the immune response in the neuronal tissues of brain in which the resident immune cells-microglia-secretes pro-inflammatory factors on the activation of cytokines to scavenge the pathogenic infiltrators and minimize the damages (Jin-Tao et al., 2015). During neuroinflammation, cytokines and chemokines play a role in mediating the inflammation of the brain tissue (Geeta et al., 2013). This resident immune cells-microglia-sometimes go uncontrolled and lead to prolonged activation in the course of time, thereby releasing enormous amount of harmful neurotoxic compounds. They enhance the apoptosis of the neuronal tissues

*Corresponding Author

Karnati Pratap Reddy, Department of Zoology, Osmania University, Hyderabad,India.E-mail: pratapkreddyou@gmail.com causing neurodegeneration and neuronal death (Miguel et al., 2011). Damon et al. (2016) reported that neuronal tissue was damaged during the process of neuroinflammation. The initiation of neuroinflammation was reported due to bacterial constituents like lipopolysaccharides and cause of mechanical injury in the brain (Cristina et al., 2012). The inflammation of brain neuronal tissue results in cognitive impairment, neurodegeneration, and neurological diseases (Roisin and McManus Michael, 2017). Oxidative stress induced excessive reactive oxygen species release, elicit cellular damage. Oxidative stress markers such as superoxide dismutase, Glutathione S-transferase, and glutathione peroxidase were altered in lipopolysaccharide-induced neuroinflammation in mice (Doaa et al., 2018). Traumatic brain injuries (TBIs) result in short- and long-term symptoms, including cognitive and emotional difficulties (Ping et al., 2013). Animals with neuroinflammation have problems to diagnose because they lack clear morphological brain deficiencies. Utagawa et al. (2008) observed the morphological changes of neurons and their 
degeneration in brain of rat exposed to neuroinflammation. Ruth et al. (2018) study showed that the neuroinflammation of brain at the neurodevelopmental stage triggered the adverse risk during the preterm birth.

The weight drop models (WDMs) are fast, reliable, and produce a significant degree of brain injury, neuroinflammation, and histological and behavioral alterations and provide important information on TBI in mice. WDM creates focal brain damage with a cortical contusion, hippocampal and thalamic damage (Niklas and Lars, 2011). The force in WDM quickly accelerates the head into a supporting foam block, resulting in diffuse brain injury without skull fracture. Primary characterization of this model revealed bilateral injury to neurons in the cerebral cortex in the brain (Thomas et al., 2004). Thus, various animal models have been developed to reproduce the neural damage, oxidative stress, and pathologic changes associated with TBI with possible underlying mechanism of neuroinflammation. Nevertheless, several of TBI models are non-physiologic or fail to duplicate the functional deficits observed in animals. In the current study, we developed a model of TBI in mice using a closed-head, weight-drop technique, which characteristically shows significant disruption to the blood-brain barrier, local and systemic inflammation, and functional deficits in behavioral, stress markers, and histological alterations. Quercetin is a potent neuroprotectant with antioxidative and anti-neuroinflammatory properties. Hence, the present study evaluates the protective effects of quercetin on neuropathological injuries of TBI in mice model which almost all equivalent to natural head injuries.

Recent studies have indicated potential of certain plants and their products such as Ashwagandha, Curcumin, and Resveratrol in treating the effects of neuroinflammation (Charbel et al., 2017; Muskan and Gurcharan, 2016; Vincenzo et al., 2018). Quercetin is a flavonoid compound and commonly found in vegetables and fruits-onions, broccoli, tomatoes, potatoes, soya beans, apples, berries, tea, and coffee and also in red wine (Alessandro et al., 2017). Quercetin is mainly present in the form of glycosides at different entities; the derivatives of quercetin are glycosides and ethers: Quercetin 3-O-galactoside, Quercetin 3-O-glucoside, Quercetin 3-O-glucuronide, Quercetin 7-O-glucoside, Quercetin 3-O-diglucoside, and Quercetin 3-methyl ether in plants (Muhammad et al., 2018). Ingested Quercetin with the help of intestinal bacteria triggers glycosidase activity, thereby hydrolyzing to form aglycone and is readily absorbed in the stomach or small intestine (Ju-Suk et al., 2016). Quercetin has got plethora pharmacological importance and known for its antioxidant activity, neurological effects, antiviral activity, anticancer activity, cardiovascular protection, antimicrobial activity, and anti-inflammatory activity (Aneela et al., 2014). Quercetin is known for its anti-inflammatory capacity which reduced the effect of $\mathrm{CCl}_{4}$-induced inflammation in liver tissues (Xi et al., 2018). Quercetin on administration decreased adipose tissues inflammation which was induced by obesity in mice (Jing et al., 2014). Recently, we have reported the neuroprotective and memory enhancing effect of quercetin by inhibiting sodium fluoride-induced neurodegeneration in developing brain of rat (Nageshwar et al., 2017). Another study also reported the antioxidant properties of quercetin against sodium-induced neurodegeneration in brain of rat (Nageshwar et al., 2018). In this study, we investigated the ameliorative effects of Quercetin as anti-inflammatory and antioxidant on neuroinflammation induced by TBI-WDM in mice through behavioral, oxidative parameters, and histological alterations.

\section{MATERIALS AND METHODS}

\section{Chemicals}

Quercetin was purchased from Sigma Aldrich Company. All other chemicals used in the investigation were of analytical grade.

\section{Animals}

The experimentation was performed using mice (Mus musculus). The animals were obtained from National Center for Laboratory Animal Sciences (NCLAS), National Institute of Nutrition (NIN), Jamai Osmania, Hyderabad-500007, Telangana, India. The protocols of the experiments were designed as per the guidelines of Departmental ethical committee, CPCSEA No: 383/01/a/CPCSE. The animals were maintained at standard laboratory conditions. Each animal was maintained in a separate polypropylene cage bedded with $2-3 \mathrm{~cm}$ paddy husk. The cage top was covered with a stainless steel grill on 12/12 hours: day/ night cycle, with $20^{\circ} \mathrm{C}-22^{\circ} \mathrm{C}$ room temperature. Water and food (Standard pellet diet NIN, NCLAS, Hyderabad) are provided through ad libitum.

\section{Quercetin dosage}

Quercetin dose $(20 \mathrm{mg} / \mathrm{kg}$ mass $)$ was standardized as appropriate dosage for animal experimentation. The quercetin (20 $\mathrm{mg} / \mathrm{kg}$ mass) dose was standardized based on the previous experimental studies (Nabavi et al., 2011; Nageshwar et al., 2017; 2018). The solvent in the ratio of $0.2 \mathrm{ml}$ of ethanol and $0.8 \mathrm{ml}$ of water for $1 \mathrm{ml}$ is used as vehicle for injection of quercetin.

\section{Experimental design}

Animals were divided into four groups.

Group-I: Mice without any treatment served as control.

Group-II: $\quad$ TBI caused by weight drop injury (WDI) as the inflammation group.

Group-III: TBI caused by WDI-inflammation along with quercetin $(20 \mathrm{mg} / \mathrm{kg}$ mass $)$ through intraperitoneal injection.

Group-IV: Mice were treated with quercetin $(20 \mathrm{mg} /$ $\mathrm{kg}$ mass) by intraperitoneal injection.

The study period was for 7 days. After 7 days, the behavioral studies were conducted and the mice were sacrificed and brains were dissected out to perform biochemical parameters and histology.

\section{Methods}

Traumatic brain injury-weight drop model

The experimental TBI-WDM in mice was maintained as per the protocol described by Marmarou et al. (1994), with necessary modifications. The procedure followed was shown in Figure 1. The metal helmet disk is $6 \mathrm{~mm}$. The weight of the 

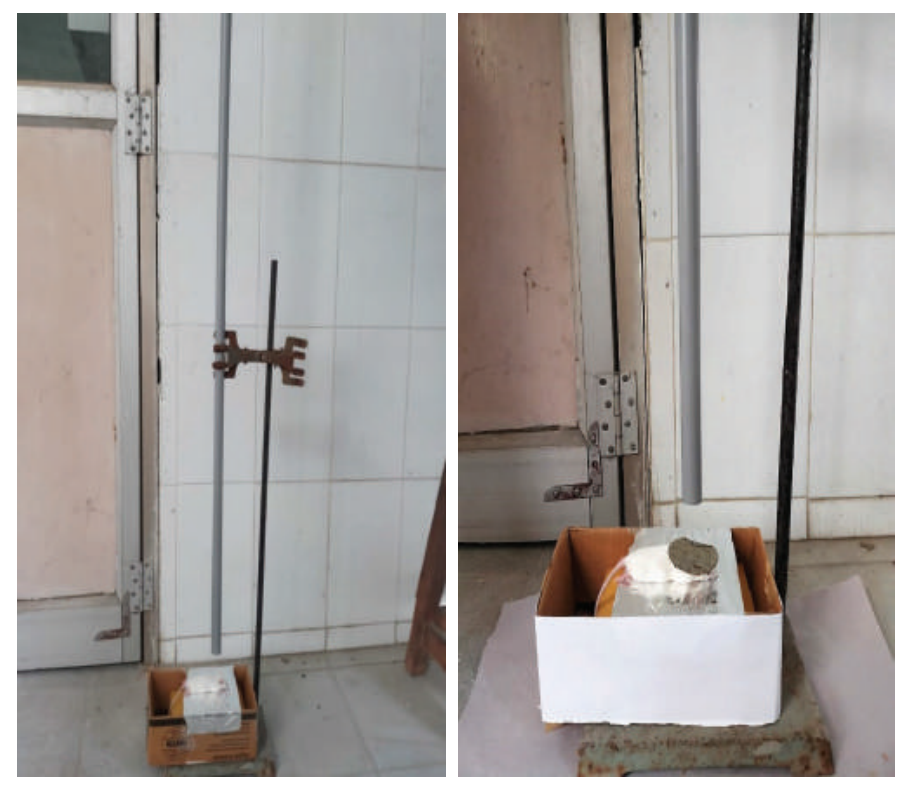

Figure 1. Traumatic brain injury-weight drop model.

brass taken was $26 \mathrm{~g}$. The mice were first anesthetized by using pentobarbital ( $40 \mathrm{mg} / \mathrm{kg}$ body weight, i.p). The anesthetized mice were brought under the vertical plastic tube and made apart about $3 \mathrm{~cm}$ in between the head of the mice against it. The metallic helmet was placed on the head of the mice and the brass weight was dropped into the plastic tube from the fixed point instantly.

\section{Behavioral studies}

\section{Rotarod test}

The rotarod test was performed according to the method of Hutter et al. (2012). The rotarod test is widely used to measure the fore and hind limb coordination, motor skills, and continues to be a primary method for the study of motor learning. The time of the instrument (Dolphin ${ }^{\mathrm{TM}}$ instruments) adjusted to 0's and the rotational speed to $20 \mathrm{rpm}$ before the experimentation. The time was noted and the results were analyzed. The results were expressed as time in seconds.

\section{Hot plate test}

The hot plate test was performed according to the method of Gunn et al. (2011). Rat's response latency to either a hind-paw lick or a jump on the hot plate (Analgesiometer-Eddy's Hot Plate) was recorded. In the absence of a response, the animals were removed from the hot plate at 60 seconds (cut off time) and a 60 seconds latency was assigned as the passive response. The results were expressed as time in seconds.

\section{Biochemical estimations}

The brain tissue was isolated and homogenized and the supernatant was collected for biochemical estimations. The lipid peroxidation was assessed by measuring thiobarbituric acid reactive substances and was expressed in terms of malondialdehyde (MDA) content, according to the method of Garcia et al. (2005). The results were expressed as nanomole $\mathrm{MDA} / \mathrm{g}$ weight of tissue. The superoxide dismutase activity was assayed according to the method of Marklund and Marklund (1974). The enzyme activity was expressed as units/mg protein. The catalase activity was assayed by the method of Aebi (1984). One unit of activity is equal to the moles of degraded $/$ minute $/ \mathrm{mg} /$ protein. The glutathione peroxidase activity was assessed by the method of Flohe and Gunzler (1984). The enzyme activity was expressed as microgram per milligram protein.

\section{Histological studies}

Golgi-Cox staining

The Golgi-Cox stock solution-fixed brain tissues were sliced at $4-10 \mu \mathrm{m}$ thickness, mounted on silanized slides, and subjected to Golgi-Cox staining according to the histological method (Gibb and Bryan, 1998). Histological changes were observed using a Lawrence \& Mayo microscope (Magnification $40 \times)$.

\section{Hematoxylin and Eosin and Congo red staining}

Brain sections were fixed in freshly prepared $10 \%$ formalin, processed routinely, and embedded in paraffin. Paraffin sections, 5- $\mu \mathrm{m}$ thick, were prepared and stained with hematoxylin and eosin (Leeson et al., 1985) and Congo red (Romhanyi, 1971) for histological examination. Sections were observed using a Lawrence \& Mayo microscope (Magnification 40×).

\section{Statistical Analysis}

The results are expressed as the mean \pm standard error of the mean (SEM). Comparison of means was conducted using one-way analysis of variance, followed by least significant difference post hoc test to compare means between the different groups. Differences were considered as significant $(p<0.05)$. Statistical analyses were performed using SPSS version 20 software (USA).

\section{RESULTS}

\section{Behavioral observations}

In the rotarod test, the experimental (inflammation) mice group showed significantly $(p<0.05)$ decreased motor coordination compared to control group. Inflammation along with quercetin group showed significantly $(p<0.05)$ improved grip strength on rotarod. The group administered with quercetin alone showed normal behavior as that of control group (Fig. 2). In the hotplate test, the paw withdrawal latency period significantly $(p<0.05)$ increased in inflammation exposed mice compared to control mice, and quercetin-treated mice showed significantly $(p<0.05)$ declined paw withdrawal latency period compared to inflammation group (Fig. 3).

\section{Biochemical assessment of oxidative stress markers}

The lipid peroxidation level was significantly $(p<0.05)$ increased in inflammation group compared with the control group. Inflammation group with quercetin significantly $(p<0.05)$ decreased the lipid peroxidation level (Fig. 4). Oxidative stress markers such as superoxide dismutase, catalase, and glutathione peroxidase activities significantly $(p<0.05)$ declined in inflammation group compared with control group. The inflammation group with quercetin significantly $(p<0.05)$ reversed superoxide dismutase, 


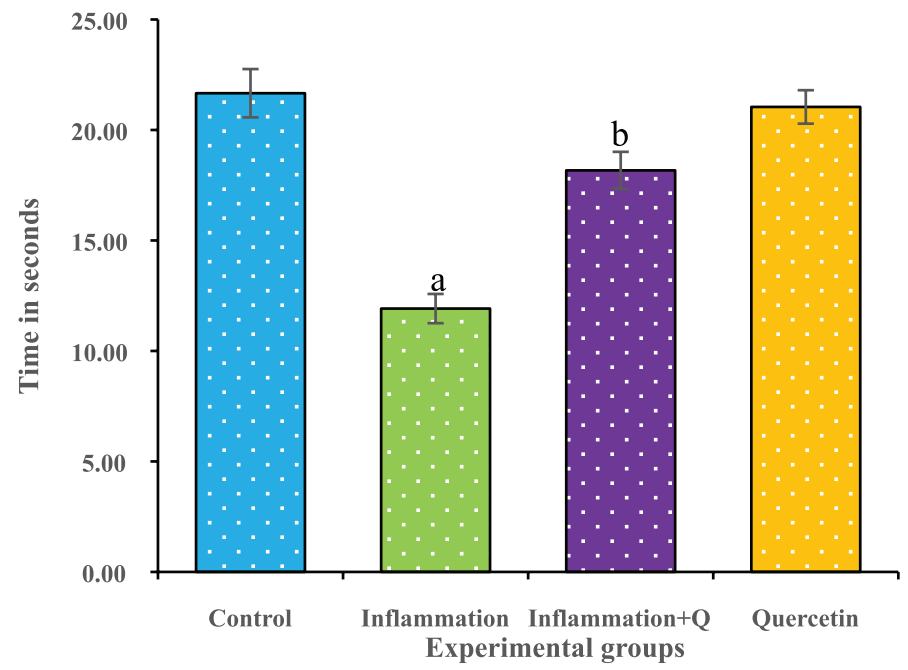

Figure 2. Effect of quercetin treatment on motor coordination (rotarod test) in mice subjected to inflammation for 7 days. ${ }^{a} p<0.05$ as compared to control group and ${ }^{\mathrm{b}} p<0.05$ as compared to inflammation group. Data expressed as the mean $\pm \operatorname{SEM}(n=5)$ and results were shown in time (second).

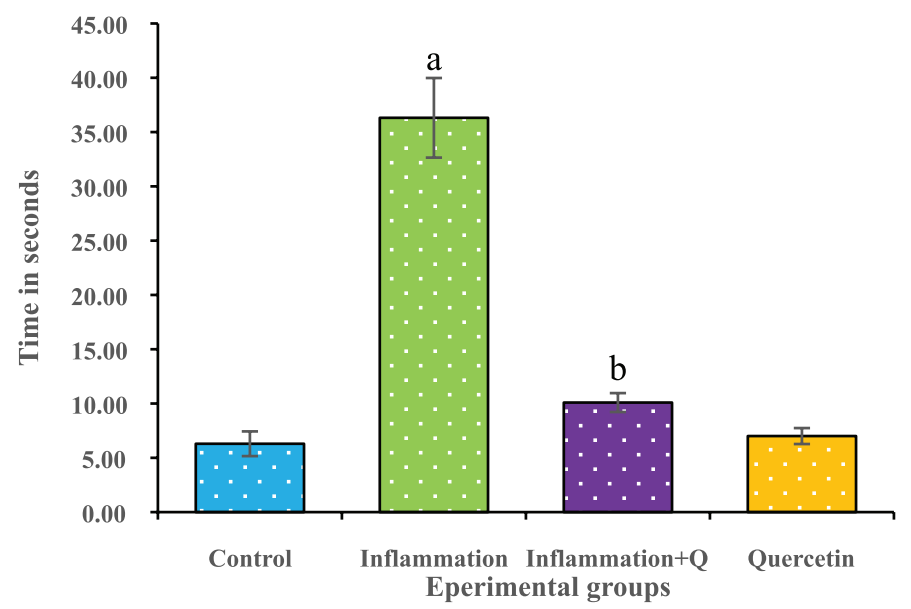

Figure 3. Effect of quercetin treatment on latency period (Hotplate test) in mice subjected to inflammation for 7 days. ${ }^{a} p<0.05$ as compared to control group and ${ }^{\mathrm{b}} p<0.05$ as compared to inflammation group. Data expressed as the mean \pm SEM $(n=5)$ and results were shown in time (second)

catalase, and glutathione peroxidase activities as compared to the inflammation group (Figs. 5-7).

\section{Histological study results}

The histological sections of inflammation and quercetin-treated mice brains were observed with Golgi-Cox, Hematoxylin-eosin, and Congo red stains in the cerebral cortex region. In the Golgi-Cox stained sections, the neuronal morphological changes such as neuron structure, density, and neuron connections were less and some of them degenerated in cerebral cortex region of the brain of inflammation group compared to the control group. This neuronal morphological damage was markedly reversed in the inflammation + Quercetin group (Fig. 8). In the Hematoxylin-eosin stained section, the size, shape of the cell, nuclear membrane, and a number of

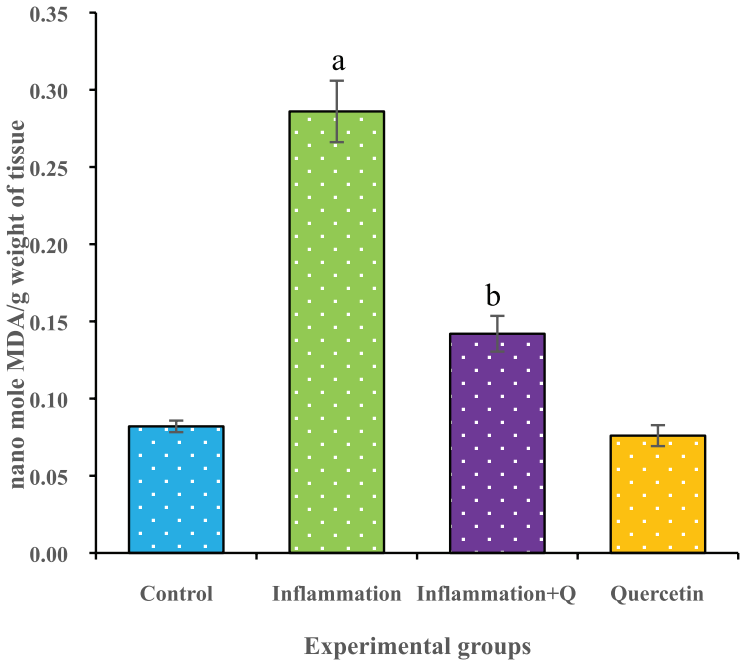

Figure 4. Effect of quercetin on lipid peroxidation levels in the brain of mice with inflammation for 7 days. Values are expressed as mean $\pm \operatorname{SEM}(n=5) .{ }^{\mathrm{a}} p<0.05$ compared with control, ${ }^{\mathrm{b}} p<0.05$ compared with the inflammation group.

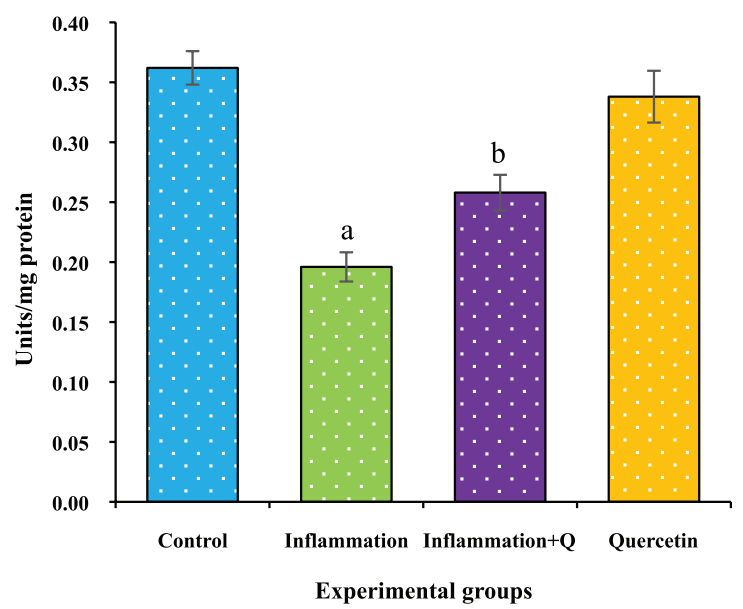

Figure 5. Effect of quercetin on superoxide dismutase activity in the brain of mice with inflammation for 7 days. Values are expressed as the mean $\pm \operatorname{SEM}(n=5) .{ }^{\mathrm{a}} p<0.05$ compared with control, ${ }^{\mathrm{b}} p<0.05$ compared with the inflammation group.

neurons were noticeably damaged in the cerebral cortex region of the brain of inflammation group. Regeneration of cellular structures, normal size, shape, and arrangement of neurons were observed in the inflammation + Quercetin group (Fig. 9). In the Congo red-stained sections, specific changes of amyloid protein plaques formation and irregular shape of neurons were detected in cerebral cortex region of the brain of inflammation group. The inflammation + Quercetin group was noticed with decreased amyloid plaque formation and clear structure of neuron (Fig. 10) while the morphology of neurons in Quercetin group was same as that of control group.

\section{DISCUSSION}

Neuroinflammation, which was induced by TBI (weight drop model), resulted in alteration of the motor and 


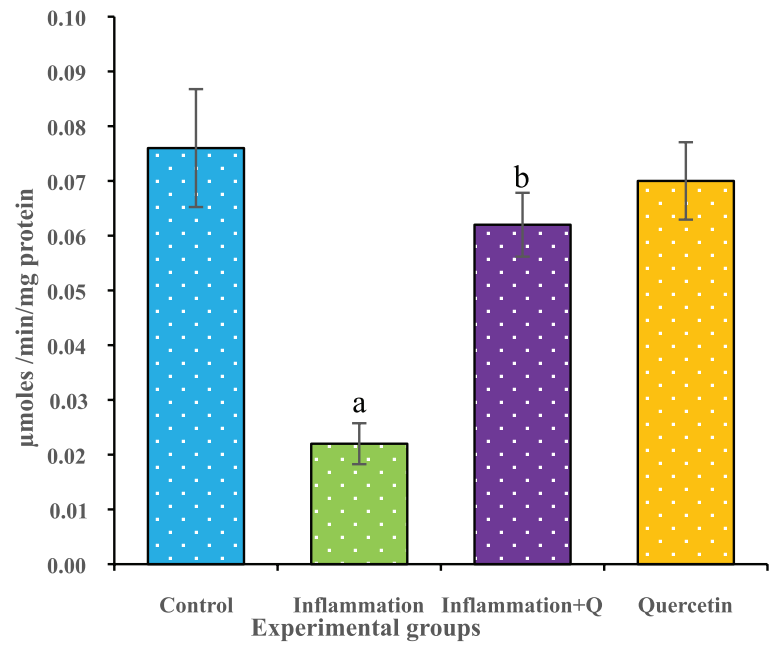

Figure 6. Effect of quercetin on catalase activity in the brain of mice with inflammation for 7 days. Values are expressed as the mean \pm SEM $(n=5) .{ }^{\mathrm{a}} p<0.05$ compared with control, ${ }^{\mathrm{b}} p<0.05$ compared with the inflammation group.

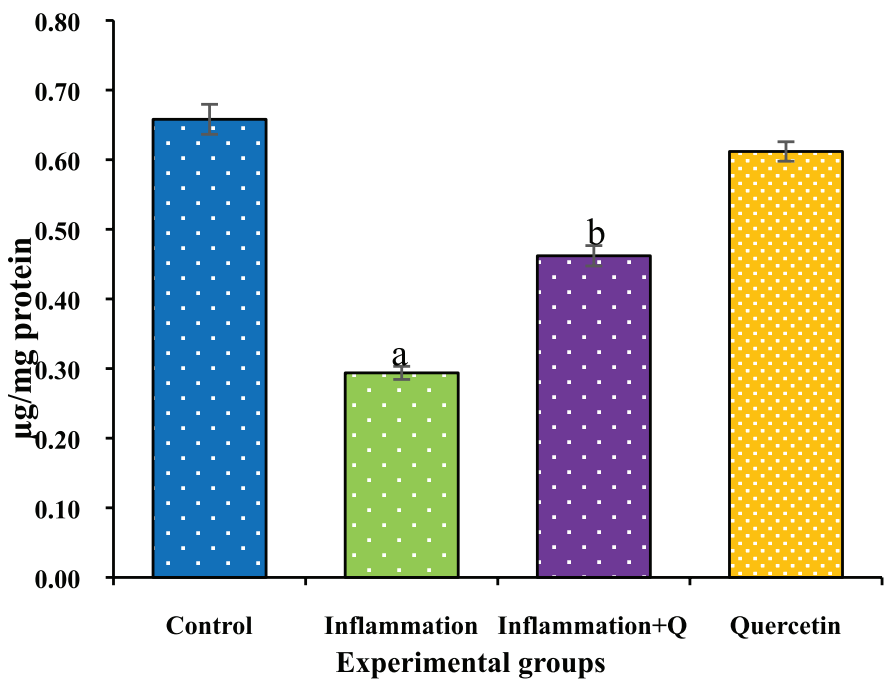

Figure 7. Effect of quercetin on glutathione peroxidase activity in the brain of mice with inflammation for 7 days. Values are expressed as the mean \pm SEM $(n=5) .{ }^{\mathrm{a}} p<0.05$ compared with control, ${ }^{\mathrm{b}} p<0.05$ compared with the inflammation group.

nociceptive behavioral activities. The course of inflammation is associated with motor and cognitive deficits (Rachel and Justin, 2013). Jin-Song et al. (2017) study suggested that motor coordination was decreased in rats by 1-Methyl-4-phenyl-1, 2, 3, 6-tetrahydropyridine-induced neuroinflammation. The inflammation in the brain also gives chances for neurotoxicity, which usually brought death to the cells (Daniela et al., 2018). The present study indicates that the inflammation group treated with quercetin showed an improvement in their ability to manage themselves along with the rod rotations and also in their nociceptive capacity to respond to the thermal heat exerted by Eddy's hot plate when compared to the inflammation subjected group. The inflammation subjected group lost their ability to grip during rod rotations for which is required to manage from falling
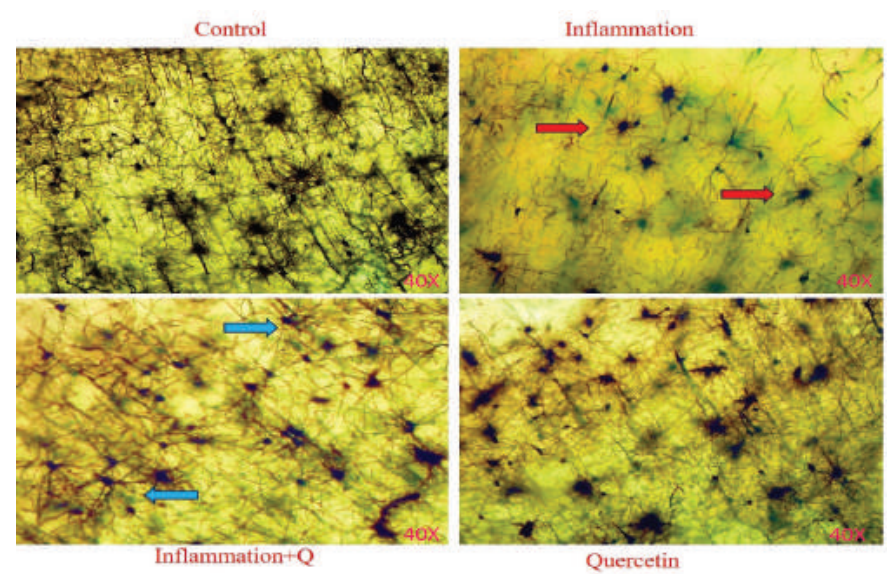

Figure 8. Quercetin effects on neuronal morphology and connections caused by TBI-WDI observed after 7 days. Density of neural connections and dendrites were clear in Control, Inflammation + Quercetin, and Quercetin groups (blue arrows); degenerated neurons with irregular shape was distinct in inflammation group compared to the control group (red arrows) (Golgi-Cox stain, Magnification-40×).

off and also do not responds to thermal heat when compared to the control group. This refers the efficiency of quercetin against motor, cognitive, and nociceptive responsive alterations.

In the neuroinflammation process, oxidative stress is involved in numerous identified pathological mechanisms ending in neuronal cell death. Federico et al. (2015) reported excessive production of reactive oxygen species and nitrogen oxygen species due to the beginning, and progression of neurodegenerative diseases. Tamy et al. (2009) also reported that continuing neuroinflammation contains not only long-standing activation of microglia and subsequent sustained release of inflammatory mediators but also outcomes in increased oxidative stress. The previous findings reported that lipid peroxidation was gradually increased during tissue inflammation (Somayeh et al., 2018). Several studies have suggested that free radical triggered peroxidative events occur after TBI (Tao et al., 2014). Neuroinflammation treated mice had significant reductions in activity of antioxidant enzymes such as superoxide dismutase, catalase, and glutathione peroxidase (Luisa et al., 2018). The increased lipid peroxidation was observed in injury-induced inflammation may be due to increased release of inflammatory mediators, as well as oxidative stress. Furthermore, reversal of lipid peroxidation levels and the activity of antioxidant enzymes such as superoxide dismutase, glutathione peroxidase, and catalase were may be due to treatment with the quercetin. Quercetin is the most powerful flavonoid for protecting the body against reactive oxygen species, produced during the normal metabolism (Liu et al., 2012). One of the most important mechanism and the sequence of events by which free radicals interfere with the cellular functions is lipid peroxidation, which eventually leading to the cell death. To inhibit this cellular damage from reactive oxygen species, living organisms have developed antioxidant line of defense systems. These include enzymatic and non-enzymatic antioxidants that keep in check reactive oxygen species or reactive nitrogen species level and repair oxidative cellular injury (Nabavi et al., 2011). The major enzymes, directly involved in the neutralization of 

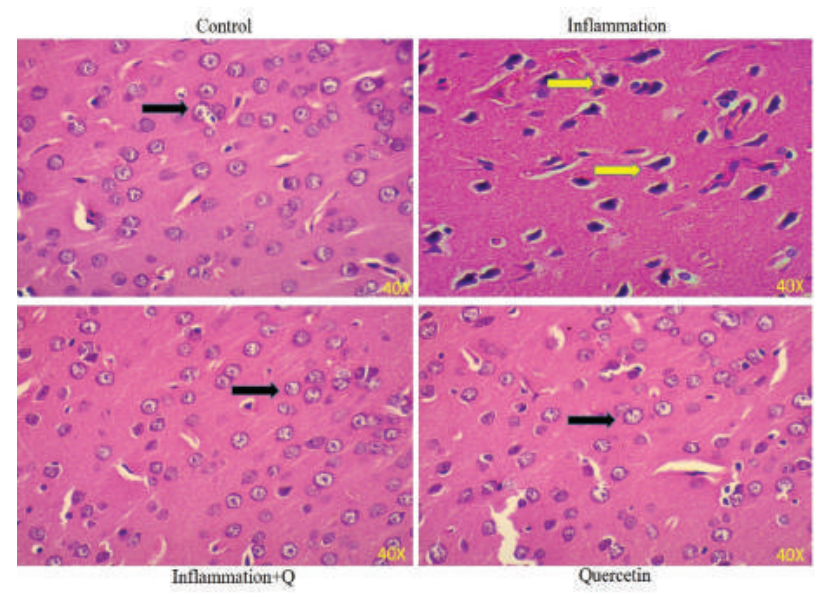

Figure 9. Quercetin effects on neuron shape, cell membrane, and neurons arrangement caused by TBI-WDI observed after 7 days. Inflammation group: showing the cells with irregular shape and indistinct nuclear membrane in the cerebral cortex region of the brain (Yellow arrows); Control, Inflammation + Quercetin, and Quercetin groups showing the normal cells with regular shape and size in the cerebral cortex region of the brain (H\&E stain, Magnification-40×).

reactive oxygen species/reactive nitrogen species are glutathione peroxidase, superoxide dismutase, and catalase. Quercetin, the antioxidant, prevents the oxidation chain initiation and stops chain propagation (Pany et al., 2014). Quercetin not only acts as an antioxidant with the ability of direct hydrogen-donating properties to quench reactive oxygen species but also may exert modulatory actions on the endogenous anti-oxidative defense system through interacting with intracellular signaling cascades (Chan-Min et al., 2015).

The implementation of WDM to mice in the present study has showed the loss of neurons, impairment of nociceptive stimuli, grip strength, and oxidative metabolism. The microglial neuroinflammation also produces reactive oxygen species and nitric oxide (Andrew and Jean, 2011), thereby causing oxidative stress as a sensitive figure in the brain tissues (Valerio et al., 2016), taken together leading to axonal loss. This may be due to the elevated levels of apoptotic markers and cognitive impairment as observed with behavioral changes. Liang et al. (2014) reported that brain tissue exhibited severe structural abnormalities, a vacuolated fibrous structure, neural cell shrunken, and neuron degeneration in TBI model in rat. Another experimental study demonstrated the areas of injury on the olfactory bulbs and inferior surface of the frontal lobes in brain of rat associated with TBI (Sung et al., 2013). Present study also showed that inflammation mice group showed fewer neural connections, amyloid protein plaque formation, and indistinct shape and size of neurons compared to the control group. The quercetin treatment has showed the reversal of these changes providing evidence of its efficiency to ameliorate neurodegeneration.

Quercetin anti-inflammatory effects involve numerous pathways. Quercetin can suppress the activity of cellular proteins involved in inflammatory response and impede $\mathrm{NF} \kappa \mathrm{B}$ transcription factor that controls the expression of proinflammatory molecules (Federico, 2012). Quercetin exerts inflammatory activity by inhibition of a number of different
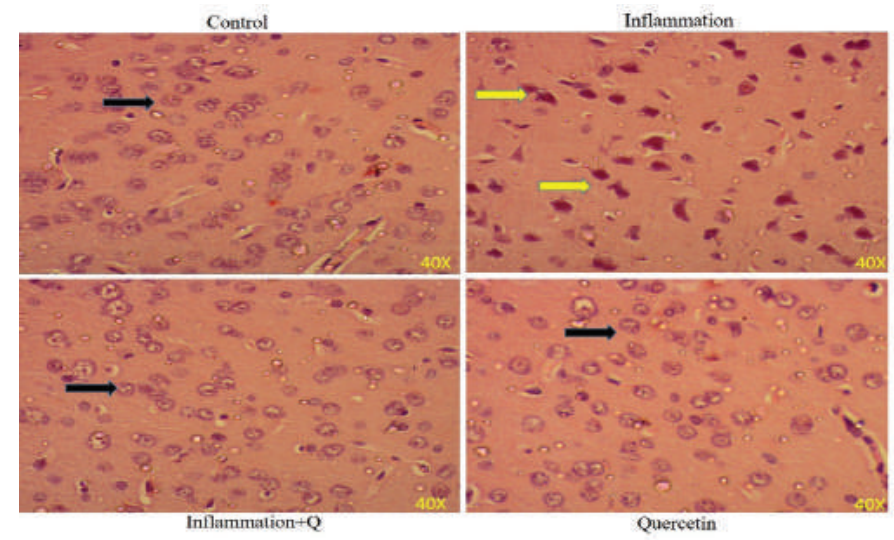

Figure 10. Quercetin effects on Congo red stain specific changes in amyloid protein plaques formation, neuron shape, and neurons arrangement caused by TBI-WDI observed after 7 days. Yellow colored arrow mark showing the amyloid protein plaques formation, cells with irregular shape and nuclear membrane was indistinct in the cerebral cortex region of inflammation group and black colored arrow mark showing the decreased amyloid protein plaques formation, closely arranged neurons, circular shape, and nuclear membrane was clear in the cerebral cortex region of control, Inflammation + Quercetin, and Quercetin groups (Congo red stain, Magnification-40×).

molecules that play an important role in inflammation and acts as an inhibitor for cyclooxygenase, 5-lipoxygenase, and glutathione S-transferase (Russo et al., 2012). Quercetin modulate both the lipooxygenase and cyclooxygenase pathways at relatively lower concentrations, while at high concentrations, the lipooxygenase pathway was the primary target of inhibitory anti-inflammatory activity (Natarajan et al., 2016). Activation of microglia and astrocytes associated with neurons induces the expression of proinflammatory mediators such as cytokines, inducible nitric oxide synthase, and nitric oxide; these leads to neuronal degeneration (Lyman et al., 2014). Quercetin ability to modulate nitric oxide production, glial signaling cascades, and pro-inflammatory cytokines might suppress neuro-inflammation, thereby inhibiting neuronal death (Lucio et al., 2016). Quercetin antagonizes microglia activation and reduces the release of proinflammatory cytokines that would be of ample relevance (Entaz et al., 2017). Quercetin, through its direct free radical scavenging and metal chelating actions, can effectively inhibit oxidative stress-mediated behavioral and histological alterations in inflamed mice. Therefore, this study provides an evidence for quercetin potential on reversing the altered motor coordination, nociceptive sensitivity, oxidative stress, and neurodegeneration in TBI model of neuroinflammation.

\section{CONCLUSION}

In conclusion, the study demonstrated multifaceted effects of quercetin on functional recovery in WDI model-induced neuroinflammation associated with behavioral, biochemical, and histological alterations. The study indicated that antioxidant property and blockade of inflammatory mediators production by quercetin treatment displayed potent neuroprotective effect. Hence, quercetin has potent protective ability against WDI model-induced neuroinflammation in mice. However, a potential limitation of our study is that we did not observe animals for long-term functional outcomes, such as oxidative stress, behavioural, and 
histological deficits along with molecular mechanisms and their amelioration. Furthermore, limitation of the TBI model includes some variability and it may be, at least initially, technically challenging with marked differences in outcome. Even minor changes in craniotomy position may result in large differences in biochemical, behavioral, and histological outcomes. Furthermore, elaborate studies are required to record long-term changes, its molecular mechanism, and potential ameliorative target for quercetin for head injury neural damages.

\section{ACKNOWLEDGMENTS}

Authors acknowledge the partial funding under UGC-SAP-DSA (F.5-26/2015/DSA-I (SAP-II), program of Department of Zoology, Osmania University.

\section{CONFLICT OF INTEREST}

Authors declare no conflict of interest.

\section{REFERENCES}

Aebi H. Catalase in vitro. Meth Enzymol, 1984; 105:121-6. Alessandro M, Olga B, Daniele R, Tatiana B, Gianni S, Massimo $\mathrm{T}$, Carmela DR. Research progress in the modification of quercetin leading to anticancer agents. Molecules, 2017; 22:1-27.

Andrew DK, Jean HG. Features of microglia and neuroinflammation relevant to environmental exposure and neurotoxicity. Int J Environ Res Public Health, 2011; 8:2980-3018.

Aneela M, Farhan AK, Amara M, Adeem M, Saira A, Muhammad A, Sabiha K, Yasir A, Imran T. Pharmacological applications of quercetin and its derivatives: a short review. Trop J Pharm Res, 2014; 13(9):1561-6.

Chan-Min L, Jie-Qiong M, Wan-Ru X, Si-Si L, Zhao-Jun F, Gui-Hong Z, Ai-Min W. Quercetin protects mouse liver against nickel-induced DNA methylation and inflammation associated with the Nrf2/HO-1 and p38/ STAT1/NF-kB pathway. Food Chem Toxicol, 2015; 82:19-26.

Charbel M, Michaeline H, Xu H, Jaeil A, Robert A, Rissman PS, Aisen Scott TR. Resveratrol regulates neuro-inflammation and induces adaptive immunity in Alzheimer's disease. J Neuroinflamm, 2017; 14(1):1-10.

Cristina P, Maria PG, Elena G, Luisa GC, Antonia G, Javier V, Angelica C, Rosa MR, Diego R. Lipopolysaccharide-induced neuroinflammation leads to the accumulation of ubiquitinated proteins and increases susceptibility to neurodegeneration induced by proteasome inhibition in rat hippocampus. J Neuroinflamm, 2012; 9(87):1-10.

Damon JD, Ning Q, Jonathan PG. Neuroinflammation: the devil is in the details. J Neurochem, 2016; 139:136-53.

Daniela SA, Emilia A, Gaviglio JM, Peralta R, Claudio B, Maria PA, Liliana M, Cancela PI. Phosphatidyl-Inositol-3 kinase inhibitors regulate peptidoglycan-induced myeloid leukocyte recruitment, inflammation, and neurotoxicity in mouse brain. Fronti Immunol, 2018; 9:1-14.

Doaa AE, Ahmed M, Nada S, Amany E, Sanaa K Trans-cinnamaldehyde modulates hippocampal Nrf2 factor and inhibits amyloid beta aggregation in LPS-induced neuroinflammation mouse model. Neurochem Res, 2018; 43:2333-42.

Entaz B, Ji-Ye K, Hyonok Y. Quercetin attenuates manganese-induced neuroinflammation by alleviating oxidative stress through regulation of apoptosis, iNOS/NF- $\mathrm{kB}$ and $\mathrm{HO}-1 / \mathrm{Nrf} 2$ pathways. Intern J Mol Sci, 2017; 18:1-19.

Federico D. Life or death: neuroprotective and anticancer effects of quercetin. J Ethanopharmacol, 2012; 143:383-96.

Federico D, Juan AA, Florencia A, Fernanda B, Carolina E, Marcela M, Felicia R, Lucia V. Quercetin in brain diseases: potential and limits. Neurochem Intern, 2015; 89:140-8.

Flohe L, Gunzler WA. Assays of glutathione peroxidase. Meth Enzymol, 1984; 105:114-21.
Garcia YJ, Rodriguez-Malaver AJ, Penaloza N. Lipid peroxidation measurement by thiobarbituric acid assay in rat cerebellar slices. J Neurosci Methods, 2005; 144(1):127-35.

Geeta R, Andrew GM, Mario TP. Cytokines and chemokines at the crossroads of neuroinflammation, neurodegeneration, and neuropathic pain. Mediat Inflamm, 2013; 2013:1-20.

Gibb R, Bryan K. A method for vibrotome sectioning of Golgi-Cox stained whole rat brain. J Neurosci Methods, 1998; 79:1-4.

Gunn A, Bobeck EN, Weber C, Morgan MM. The influence of nonnociceptive factors on hot-plate latency in rats. J Pain, 2011; 12(2):222-7.

Hutter SJA, Gendelman HE, Mosley RL. Murine motor and behavior functional evaluations for acute 1-methyl-4-phenyl-1,2,3,6-tetrahydropyridine (MPTP) intoxication. J Neuroimm Pharmacol, 2012; 7(1):279-88.

Jing D, Xian Z, Lei Z, Hui-Xi B, Na X, Bin B, Jian L. Quercetin reduces obesity associated ATM infiltration and inflammation in mice: a mechanism including AMPK $\alpha 1 /$ SIRT1. J Lipid Res, 2014; 55(3):363-74.

Jin-Song Y, Xiao-Hong W, Hao-Gang Y, Li-Song T. Tangeretin inhibits neurodegeneration and attenuates inflammatory responses and behavioural deficits in 1-methyl-4-phenyl-1,2,3,6-tetrahydropyridine (MPTP)-induced Parkinson's disease dementia in rats Inflammopharmacology, 2017; 25:471-84.

Jin-Tao L, Mo-Han D, Jie-Qiong Z, Ya B, Fang K, Liang-Wei C. Microglia and astroglia: the role of neuroinflammation in lead toxicity and neuronal injury in the brain. Neuroimmunol Neuroinflamm, 2015; 2(3):131-7.

Ju-Suk N, Ashish RS, Lich TN, Chiranjib C, Garima S, Sang-Soo L. Application of bioactive quercetin in oncotherapy: from nutrition to nanomedicine. Molecules, 2016; 108(21):1-23.

Leeson CR, Leeson TS, Paparo AA. Text book of histology. 5th edition, WB Saunders Company, USA, 1985.

Liang W, Yanfei Z, Cheng Y, Qi W, Zhongwei Z, Zhiyang $S$. Neuroprotective effects of ebselen in traumatic brain injury model: involvement of nitric oxide and p38 mitogen-activated protein kinase signalling pathway. Clin Exp Pharmacol Physiol, 2014; 41:134-8.

Liu H, Zhang L, Lu S. Evaluation of antioxidant and immunity activities of quercetin in isoproterenol-treated rats. Molecules, 2012; 17:4281-91.

Lucio GC, Jacqueline MG, Pamela JR, Claudia P. Mechanisms of neuroprotection by quercetin: counteracting oxidative stress and more. Oxid Med Cell Longev, 2016; 2016:1-10.

Luisa C, Maria SS, Rafaela C, Rosa C, Lucia I, Arianna M, Giovanna L, Susanna I. Short-term fructose feeding induces inflammation and oxidative stress in the hippocampus of young and adult rats. Mol Neurobiol, 2018; 55:2869-83.

Lyman M, Lloyd DG, Ji X, Vizcaychipi MP, Ma D. Neuroinflammation: the role and consequences. Neurosci Res, 2014; 79:1-12.

Marklund S, Marklund G. Involvement of the superoxide anion radical in the autoxidation of pyrogallol and a convenient assay for superoxide dismutase. Eur J Biochem, 1974; 47(3):469-74.

Marmarou A, Montasser A, Abd-elfattah F, van den BW, Campbell J, Kita H, Demetriadou K. A new model of diffuse brain injury in rats Part I: pathophysiology and biomechanics. J Neurosurg, 1994; 80:291-300.

Miguel A, Burguillos T, Deierborg EK, Annette P, Nabil H, Albert GQ, Josefina C, Patrik B, Elisabet E, Jose LV, Bertrand J. Caspase signalling controls microglia activation and neurotoxicity. Nature, 2011; 472:319-23.

Muhammad FM, Ahsan M, Abdul W. Quercetin - a mini review. Mod Concep Dev Agrono, 2018; 1(2):1-5.

Muskan G, Gurcharan K. Aqueous extract from the with aniasomnifera leaves as a potential antineuroinflammatory agent: a mechanistic study. J Neuroinflamm, 2016; 193(13):1-17. 
Nabavi SF, Moghaddan AH, Nabavi SM, Eslami S. Protective effects of curcumin and quercetin on thyroid function in sodium fluoride intoxicated rats. Fluoride, 2011; 44(3):147-52.

Nageshwar M, Kirankumar N, Rajkiran RB, Chandrashakar RN, Pratap RK. Quercetin treatment against $\mathrm{NaF}$ induced oxidative stress related neuronal and learning changes in developing rats. J Kin Saudi Univ Sci, 2017; 29:221-9.

Nageshwar M, Sudhakar K, Pratap RK. Quercetin ameliorates oxidative stress, neural damage of brain and behavioral impairment of rat with fluoride exposure. Intern J Pharmacol Sci Res, 2018; 9(8):1000-10.

Natarajan S, Kasi PD, Seyed FN, Nady B, Seyed MN. Bioactive effects of quercetin in the central nervous system: focusing on the mechanisms of actions. Biomed Pharm, 2016; 84:892-908.

Niklas M, Lars H. Animal modelling of traumatic brain injury in preclinical drug development: where do we go from here? Br J Pharmacol, 2011; 164:1207-29.

Pany S, Abhisek P, Sahu PK. Neuroprotective role of quercetin in neurotoxicity induced rats: role of neuro-inflammation in neurodegeneration. Asian J Pharm Clin Res, 2014; 40:178-83.

Ping L, Yong-sheng L, David Q, Allal B, Yong J. Inhaled nitric oxide improves short term memory and reduces the inflammatory reaction in a mouse model of mild traumatic brain injury. Brain Res, 2013; 1522:67-75.

Rachel AK, Justin SR. Neurogenesis, inflammation and behavior. Brain Behav Immun, 2013; 27:22-32.

Roisin M, McManus Michael TH. Role of neuroinflammation in neurodegeneration: new insights. Alzheimer's Res Ther, 2017; 14(9):1-7.

Romhanyi G. Selective differentiation between amyloid and connective tissue structures based on the collagen specific topo-optical staining reaction with Congo red. Virchows Arch, 1971; 354:209-22.

Russo M, Spagnuolo C, Tedesco I, Bilotto S, Russo GL. The flavonoid quercetin in disease prevention and therapy: facts and fancies. Biochem Pharmacol, 2012; 83(1):6-15.

Ruth G, Rob JJ, Westerlaken Daan R, Ophelders MG, Alan H, Jobe MW, Kemp SG, Kallapur LJ, Zimmermann PT, Sangild SP, Pierre G, Boris W, Kramer BF, Tim GW. Chorioamnionitis, neuroinflammation, and injury: timing is key in the preterm ovine fetus. J Neuroinflamm, 2018; 113(15):1-13.

Somayeh K, Adele J, Parvin B. Kaempferol attenuates cognitive deficit via regulating oxidative stress and neuroinflammation in an ovariectomized rat model of sporadic dementia. Neur Regenerat Res, 2018; $1827-32$.

Sung HY, Josh G, Matt G, David S, Rebecca S, Timothy AP, Michael DG, Daniel GR, Alex BL. A murine model of mild traumatic brain injury exhibiting cognitive and motor deficits. J Surg Res, 2013; 184:981-8.
Tamy CF, Laura TA, Fiona EMA, Malu GT. Does neuro inflammation fan the flame in neurodegenerative diseases. Mol Neurodeg, 2009; 47(4):1-13.

Tao Y, Bin K, Jian-Wen G, Yong-Qin K, Lin C, Wen-Tao Y, Xun $\mathrm{X}$, Hai-Feng S. Anti-apoptotic and anti-oxidative roles of quercetin after traumatic brain injury. Cell Mol Neurobiol, 2014; 34:797-804.

Thomas M, Hallam C, Floyd MM, Folkerts L, Lee QZ, Gong BG, Lyeth J, Paul M, Robert FB. Comparison of behavioral deficits and acute neuronal degeneration in rat lateral fluid percussion and weight drop brain injury models. J Neurotrauma, 2004; 21(5):521-39.

Utagawa A, Truettner JS, Dietrich WD, Bramlett HM. Systemic inflammation exacerbates behavioral and histopathological consequences of isolated traumatic brain injury in rats. Exp Neurol, 2008; 211:283-91.

Valerio C, Antonio O, Mauro M. Is modulation of oxidative stress an answer? The state of the art of redox therapeutic actions in neurodegenerative diseases. Oxid Med Cell Longev, 2016; 1-11.

Vincenzo S, Gabriella C, Stefania S, Stefano D, Francesca C, Andrea P, Pietro G, Morena Z. Curcumin prevents acute neuroinflammation and long-term memory impairment induced by systemic lipopolysaccharide in mice. Front Pharmacol, 2018; 9:1-12.

Xi L, Qianwen J, Qunyan Y, Beili X, Lixin L, Shuncai Z, Chuantao T. The flavonoid quercetin ameliorates liver inflammation and fibrosis by regulating hepatic macrophages activation and polarization in mice. Front Pharmacol, 2018; 9:1-14.

\section{How to cite this article:}

Kom HH, Nageshwar M, Srilatha K, Reddy KP. Protective effect of quercetin on weight drop injury model-induced neuroinflammation alterations in brain of mice. J Appl Pharm Sci, 2019; 9(04):096-103. 